Baltic Astronomy, vol.0, 00-00, 1996.

Proc. Internat. Cooperation in Dissemination of Astronomical Data, July 3-5, 1996, St.-Petersburg, Russia

\title{
PREPARING A PUBLIC DATABASE OF RADIO SOURCES
}

\author{
H. Andernach ${ }^{1}$, S.A. Trushkin ${ }^{2}$, A.G. Gubanov ${ }^{3}$, O.V. Verkhodanov ${ }^{2}$, \\ V.B. Titov ${ }^{3}$ and A. Micol ${ }^{4}$ \\ ${ }^{1}$ IUE Observatory, Villafranca, Apdo. 50727, E-28080 Madrid, Spain \\ ${ }^{2}$ Special Astrophysical Observatory RAS, Nizhnij Arkhyz, 357147 Russia \\ ${ }^{3}$ Astronomical Inst., St.-Petersburg State University, 198904 Russia \\ ${ }^{4}$ ST-ECF, Karl-Schwarzschild-Str. 2, D-85748 Garching, Germany
}

Received July 20, 1996.

\begin{abstract}
We have collected the largest existing set of radio source lists in machine-readable form : 320 tables with 1.75 million records. Only a minor fraction of these is accessible via public databases. We describe our plans to make this huge amount of heterogeneous data accessible in a homogeneous way via the World Wide Web, with reliable cross-identifications, and searchable by various observables.
\end{abstract}

Key words: radio sources: general; databases; catalogues

Surveys of radio sources have always been instrumental in widening our horizon of the distant Universe: optical identifications of the sources are pointing us to the most distant galaxies known, and the perfect isotropy of the source distribution in even the deepest surveys shows that we are sampling a much larger volume of space than we do with magnitude-limited galaxy surveys. Of course, an efficient search for a certain type of objects (e.g. distant galaxies, variable sources, etc.) requires the filtering of large samples of radio sources carefully selected from literature by very specific criteria. Until now this required to construct these various samples by tedious merging and sorting of numerous individual source catalogues. In addition, existing astronomical databases show a notable lack in published information on radio sources, and data centers provide only a small part of the largest published source catalogues.

One of us (Andernach 1990) has noted this lack years ago. Since then he has gathered the yet most complete set of radio source catalogues in electronic form : 320 source tables with a total of 1.75 million entries. 
Only a minor fraction of the tables is accessible through public databases. This collection is the result of six years of persistent requests to the authors, even though in an early email campaign radio astronomers were invited to contribute their electronic source tables to the astronomical data centres voluntarily. In an effort to recover also the largest published source lists not available in electronic form, H.A. employed a scanner and "Optical Character Recognition" (OCR) software. Since early 1995 over 100 printed data tables with $>50,000$ entries into electronic form. Staff at Special Astrophysical Observatory of the Russian Academy of Sciences (SAO RAS), also helped correcting, editing and proof-reading of the raw OCR result, as well as in the manual retyping of many source tables which were unrecoverable with OCR. Tables published only on microfiche are the most difficult ones to recover, since microfiche copiers have turned into an extremely rare species of apparatus.

Independently, SAO RAS and its St.-Petersburg branch (SPbB SAO) and the Astronomical Institute of the St.-Petersburg State University (AI $\mathrm{SPbU}$ ) maintain a collection of astronomical catalogues. The database of "Radio Astronomical Catalogues" (RAC) has been created at AI (Gubanov \& Titov 1996; http://www.aispbu.spb.su/WWW/RAC.html). The "CATalogue support System" (CATS) is being developed at SAO (Verkhodanov \& Trushkin 1995; telnet ratan.sao.ru, login as cats, $\mathrm{pw}=<\mathrm{CR}>$ ). Both are supported by the Russian Foundation for Basic Research (RFBR).

A. Micol is involved in the use of relational databases for astronomical applications. He will be responsible for the design of the database structure and its user interface, translation of user to software requirements, configuration and maintenance of a WWW server, and for application Program Interfaces (API) to commercial database management systems.

The present project joins the efforts of these groups to establish the first reasonably complete and publicly accessible database of radio sources. During the first half year of active collaboration over fifty tables and catalogues were either scanned or typed, proof-read and included in the full collection of data sets. Tools were developed to cross-identify sources from different catalogues and to construct and display their radio spectra.

Virtually all catalogues have a different format and list different observables. It will be a major challenge to provide uniform access to such a heterogeneous collection of data sets involving different methods, notations and units. Only a clear understanding of observing techniques and data-processing for a given catalogue will guarantee reliable cross-IDs between catalogues. Thus, documentation files in an agreed format will be prepared for all catalogues accessible to the search. Many catalogues 
provide only source names and will have to be completed with positional data. For other tables lacking positional errors we will insert these errors from formulae given in the publication.

The concept of a "Reference Directory" (a central repository of metadata like the descriptions of catalogue fields, their physical units, mapping of original field names to the actual name in the database, etc), will be used for catalogue browsing and to process user queries.

The basic user requirements of the proposed database consist of

- freely accessible via telnet and World Wide Web;

- inclusion of a sufficiently complete number of source catalogues with on-line documentation of their columns;

- search by position in $(\alpha, \delta)_{1950},(\alpha, \delta)_{2000}$ and Galactic coordinates;

- SQL-type query by user-specified parameters like e.g. name, flux at a given frequency, source size, spectral index, identification;

- X-window protocols for graphical tools like spectral plots, radio-sky finding charts and display of two-dimensional FITS files.

The proposed database will fill a long-standing gap in multi-waveband astronomical information systems at a time when several large-area surveys of high sensitivity and resolution are in progress with the VLA and the WSRT, producing millions of further sources. Comparison of available radio data with these new surveys will be crucial for statistical studies and undoubtedly lead to the discovery of new source populations.

Cross-identification and spectral classification of many 10,000 radio sources in our Galaxy and in extragalactic space will provide an unprecedented basis for radioastronomical and cosmological studies. Scientific applications of such a database are extremely numerous, like e.g. the search for high-redshift radio galaxies, the study of influence of a cluster environment on radio sources, the selection of targets for future space VLBI mission of extremely high angular resolution, and the selection of spectrally peculiar or time-variable sources for follow-up studies. A much better separation of the complex mix of source populations in the Galactic plane will also be possible. The project will have its educational impact on students preparing catalogue documentation and user's guides, and collaborating in specific research projects.

\section{REFERENCES}

Andernach H. 1990, Bull. Inf. CDS, 38, 69

Gubanov A.G. \& Titov V.B. 1996, Vestnik St.-Petersburg Univ., Ser. 1, Vyp. 3 (N 15), in press

Verkhodanov O.V. \& Trushkin S.A. 1995, Preprint 106 SAO RAS, p. 66 